\title{
Transformation of Nonlinear Mixture Chopped Stochastic Program Model
}

\author{
Togi Panjaitan ${ }^{1}$, Iryanto Iryanto ${ }^{2}$ \\ ${ }^{1}$ Mathematic Education Department, Faculty of Mathematic and Science, State University of Medan, Medan, Indonesia \\ ${ }^{2}$ Mathematic Department, North Sumatra University, Medan, Indonesia
}

Email address:

togipanjaitan2014@gmail.com (Togi Panjaitan)

\section{To cite this article:}

Togi Panjaitan, Iryanto Iryanto. Transformation of Nonlinear Mixture Chopped Stochastic Program Model. Applied and Computational Mathematics. Vol. 4, No. 2, 2015, pp. 69-76. doi: 10.11648/j.acm.20150402.16

\begin{abstract}
This paper describes a new approach to obtain the global optimization problem of nonlinear mixture chopped stochastic program model. The study focused on the issue of two-stage stochastic with the lack of nonlinearity, which is contained in the objective function and constraints. Variables in the first stage is worth a count, while the variable in the second stage is a mixture of chopped and continuous. Issues formulated by scenario-based representation. The approach used to complete the large scale nonlinear mix chopped program lifting unfounded variable value of the limit, forcing a variable-value basis chopped. Problems reduced is processed at the time of chopped variables held constant, and the changes made during discrete steps, in order to obtain a global optimal solution.
\end{abstract}

Keywords: Nonlinear Stochastic Programs, Equivalent model, Scenarios Formation

\section{Introduction}

Stochastic optimization programs related to decisionmaking with the uncertainty of the data problems of a time period to the next time period. Type of study object of this program is a random optimization problem, where the results of random data is not revealed at run time, and the decision will be optimized not have to anticipate future results (nonanticipation). It is closely related to the optimization of 'real time' which is the optimal decision 'here and now' in an environment of incomplete data (or uncertain).

Some researchers have filed completion method of chopped stochastic programs, with a different approach. In the two-stage method with recourse simple chopped, the approach based on the formation of the set of the second stage convex functions, which is the special structure of the second stage [1]. In-decomposition-based approach, where the first and second stage variables are binary, the approach is a branch-and-bound, in which the function value approaches optimality slicing the second stage, not convex for binary completion of the first phase have been obtained [2]. [3] developed a decomposition based algorithms to solve two-stage Chopped Stokastic Program (CSP) problems, emphasizing on the decomposition of chopped variables that appear in the first and second stage. [4] examines the related approach in which the reformulation-linearization technique is used in the decomposition scheme.

[5] use of scenario decomposition approach of [6] to develop a branch and bound algorithm to solve the two-stage CSP. The lower limit derived from the Lagrange dual, derived by doubling the constraints of non-anticipation. Subproblema dual Lagrange associated with the scenario and include variables and constraints of the first and second stage. Subproblema is more difficult to solve than the Benders decomposition based method. Although dual Lagrange provide strict limits, the solution requires the use of methods and results subgradien difficulty in computing. [7] also filed a branch and bound algorithm for the two-stage PSC with chopped-mix variables in the first stage and chopped-pure variables in the second stage. Reformulation done to exploit the special structure that emerged from the review matrix refixed in a two-stage CSP. Lagrange relaxation approach to solve the two-stage PSC filed by [8].

[9] proposed a finite scheme for two-stage stochastic program with discrete distribution and the second-stage variables pure chopped. For this problem, which was observed only a count value of the relevant right side parameter. This fact is used to identify the set of accounting, which is called the set of candidates, the first space-stage variable containing the optimal solution. In its basic form, 
made a complete enumeration of the set of candidates to find the optimal solution. Evaluation of an element of the set requires the completion of a second-stage chopped subproblema that relate the realization that perhaps all of the uncertain parameters. Thus, explicit enumeration of all the elements, in general, computationally impossible. Search 'neighborhood' feasible to complete the two-stage CSP has been developed by [10] completing the portfolio optimization model.

Research in dual stage Mixture Chopped Stochastic Program (MCSP) is still lacking. [11] apply heuristic algorithm in which the hedge (hedging) progressive combined with Tabu search to solve MCSP with binary variables. Mawengkang (2002) also propose a heuristic threshold price which exploit the structure of problems with the risk threshold value (value at risk) to complete the MCSP in financial optimization. [12] proposed a Lagrange relaxation for use in branch and bound algorithm to double stage MCSP. But the computational results is limited to twostage problems. [13] study the continuity of the objective function of the first stage decision, and integrating the probability measure to further reformulate two-stage MCSP to be Linear Mixture Numbers Program (LMNP), if the probability distribution of discrete and finite. Branch and Price algorithm proposed by [14] to solve the problem of dual stage Mixture Chopped Stochastic Program (MCSP) which has a special structure. [15] exploit certain substructure problems and then develop an efficient capacity planning scheme approach to solve the problem of doublestage Chopped Stochastic Program.

Sample Average Approach Method (SAAM) has been proposed by [7] to complete the stochastic program with recourse chopped. The main idea of the PRS approach to solve stochastic program are as follows: a sample $\xi^{1}, \ldots, \xi^{n}$ fromi $N$ realization of random vector $\xi(w)$ formed and consequently expectation value function $E[Q(x, \xi(w))]$ estimated by the sample mean function $N^{-1} \sum_{n=1}^{N} Q\left(x, \xi^{n}\right)$. Sample average approximation obtained, $\min _{x \in X}\left\{\hat{g}_{N}(x)=c^{T} x+N^{-1} \sum_{n=1}^{N} Q\left(x, \xi^{n}\right)\right\} \quad$ of the stochastic program then solved by deterministic optimization algorithm. This approach (and its variations) is also known by several other names such as the method of stochastic counterpart [16] and a sample trajectory optimization method of [17]. $\hat{v}_{N}$ and $\hat{x}_{N}$ denote the optimal value and optimal solution problem Sample Average Program (SAP). Then ${ }^{*}$ and ${ }_{x}^{*}$ denote the optimal value and optimal solution initial problem.

[18] proposed a method of settlement for Nonlinear Mixture Chopped Stochastic Program (NMCSP) by using "simplicial" approach. This approach is a combination of approaches based simplicial by [19] with SAP. At each iteration of the algorithm-based simplicial, SAP's procedure is applied to all subproblems stochastic linear and chopped mixture of linear stochastic problems. However the approach is not linear stochastic program must be completed which depend on the number of scenarios.
Nonlinear stochastic program presents a class of stochastic optimization problem that frequently appear in real life. Many natural systems this has no linear model pattern, resulting in non linear program methods are needed to determine optimisasinya. Another factor that look has become a fairness is uncertainty which requires stochastic program.

Sometimes the decision optimization problem thus include a variable whose value must be natural numbers, or binary $(0$ or 1). So, if given the requisite chopped against the decision variables, then the nonlinear stochastic programming model is referred to as a count of non linear stochastic program. In this study, the model studied stochastic program is Nonlinear Mixture Chopped Stochastic Program (NMCSP), which means that in addition to the required variables valuable natural numbers but there is also a variable that can be worth a continuous (fractions). This program can be used in the field of process systems engineering [20,21]. The main features of the two-stage stochastic programming model is the act of "recourse". The set of decisions is divided into two groups. A number of decisions have to be taken before the problem parameters are known: this decision is the decision of the first stage and the decision was taken in the first stage. Other decisions may be taken after the uncertainty is revealed. Recourse decision is a function of the actual realization of the uncertain parameters and the first stage of the decision. Sequence of events characterizing the model as a model recourse.

There are some things that concern the issue of two stage NMCSP, ie, convexity and continuity. This is mainly due to the requirements of chopped. If the variable is a count of only the first stage is, the nature of recourse same function as in the case of continuous. In the case of continuous nonlinear if $f$, $h$ convex and $g$ affine for all $\xi$, problema is canvex. If the chopped requirements appear in the second stage, although in the case of linear, generally recourse function is not convex. Difficulties in dimensions depending on the number of scenarios.

In connection with this, the idea of this research is to transform the model into a model of Linear Mixture Chopped Program (LMCP). This is based on uncertainty, and time can be modeled as a finite number of scenarios. Therefore the distribution function is not used the opportunity to represent uncertainty. With the distribution function, this may result in discontinuity and not convex. Likewise, the size of the equivalent model will grow very rapidly as a consequence of a number of scenarios and the amount of time horizon. To have built a method for forming an efficient number of scenarios.

\section{Method}

With the assumption of discrete probability space, resulting in the objective function can be written as a finite sum and constraints replicated for each element in $\Omega$. Suppose that $\xi$ has a discrete probability distribution on $\Omega=$ $1, \ldots, S$ with $\mathrm{P}\left(\xi=\xi_{i}\right)=\pi_{i}$. Then the problem can be written again in the form of: 


$$
\begin{aligned}
& \min f^{1}(x)+\sum_{s=1}^{S} \pi_{s} f^{2}\left(x, y, \xi_{s}\right) \\
& g^{1}(x)=0 \\
& h^{1}(x) \leq 0 \\
& h_{s}^{2}\left(x, y_{s}, \xi_{s}\right)=0 \quad \forall s=1, \cdots, S \\
& g_{s}^{2}\left(x, y_{s}, \boldsymbol{\xi}_{s}\right)=0 \quad \forall s=1, \cdots, S \\
& x \in Z_{+}^{n_{1}}, y_{s} \in Y_{s} \quad \forall s=1, \cdots, S \\
& g^{1}: R^{n_{1}} \rightarrow R^{m_{e}} \quad h^{1}: R^{n_{1}} \rightarrow R^{m_{i}} \\
& g^{2}: R^{n_{1}+n_{2}} \rightarrow R^{t_{e}} \quad h^{2}: R^{n_{1}+n_{2}} \rightarrow R^{t_{i}}, s=1, \cdots S
\end{aligned}
$$

where $\pi_{s}$ denote the probability that the scenario $s$ is accur. The deterministic equivalent formulation, is a large scale nonlinear naural number program problematic with variable $n_{1}+n_{2} s$ and $m_{e}+m_{i}+t_{e} s+t_{i} s$ is nonlinear constraints. Because of the requirements of chopped, recourse function is generally not convex and discontinuous (lower semicontinuous). Branch and Bound method, which is commonly used to resolve the problem of natural numbers linear program, can not be applied to the lower semi-continuous conditions, as there will be an infinite sub problems required. Therefore, the lower limit and the upper limit being the same, and consequently finite termination of the algorithm is not guaranteed.

Expectations in (1) includes a multi-dimensional integration. So that problems can be resolved, the uncertainty is usually expressed in a discrete distribution that is approaching. However, the need for accuracy in modeling that results in an increase in the dimensions of the optimization program. This adds to the limitations on the manner and method of modeling stochastic program completion is still at an early stage.

\subsection{Deterministic Equivalent Formulations}

Linear stochastic programming model is written as follows

$$
\left.\begin{array}{cl}
\min & g_{0}(x, \tilde{\xi}) \\
\text { s.t. } & g_{i}(x, \tilde{\xi}) \leq 0, i=1, \ldots, m, \\
& x \in X \subset \mathbb{R}^{n},
\end{array}\right\}
$$

withn $\tilde{\xi}$ is random vector which varies in the set $\Xi \subset \mathbb{R}^{k}$. More precisely, it is assumed that the family F of "events", which is a subset of $\Xi$, and distribution opportunities $\mathrm{P}$ on $\mathrm{F}$ unknown. So for each subset $A \subset \Xi$ which is the events, ie $A$ $\in F$, opportunities $\mathrm{P}$ (A) is known. Furthermore, it is assumed that the function $g_{i}(x, \cdot): \Xi \rightarrow \mathbb{R} \forall x, i$ is a random variable and distribution opportunities $\mathrm{P}$ is free. However, the problem (2) is not "well defined" for understanding "min" and also constraints unclear, if that counts is the value of a decision $x$ before knowing the realization of $\xi$. Because of the revision of the modeling process needs to be done, which will produce the equivalent deterministic models for (2).

\subsection{Determination of Scenarios}

Algorithm to solve Double Stage Linear Stochastic Program (DSLSP) is the same as in the Two-Stage Linear Stochastic Program (TSLSP), and retaining the computational capabilities. At the same time, this approach also provides asymptotic optimality. Another contribution is the proposed algorithm provides the operational realization of the notion of prolongasi which proposed first by [22]. In this study, prolongasi not only provide the basis for the results of convergence, but also provide operating policies to expand the primal completion of the initial approximation problem settlement. While prolongasi thus can not produce a decent settlement for all possible scenarios. This is taken to implement the settlement TSLSP obtained from the initial approximation problem $\mathrm{P}$. By limiting the right hand side $b(\vec{\xi})$ is random, and other data is deterministic. Also, required $b_{t}$ is affine function of $\vec{\xi}$. Completion of this fully integrated problem, resulting in each gusset $n$, , namely (i) primal decision $\bar{x}_{n}$ and (ii) dual multiplier vector $\bar{x}_{n}$. If $\vec{\xi}_{n}$ is a scenario that is associated with gusset $n$ is a scenario that is associated with gusset $n$. With $\mathcal{B}_{n}$ an optimal row to gusset Linear Program below :

$$
\left\{\begin{array}{l}
\min \left(c_{n}-\sum_{\tau=t_{n}+1} E\left[\left(A_{r t_{n}}^{T} \bar{\pi}_{\tau}\right) \mid\right]\left(\vec{\xi}_{n}\right)\right)^{T} x_{n} \\
A_{t_{n} t_{n}} x_{n}=b_{n}-\sum_{m \in H_{n}: \bar{p}_{n}>0} A_{t_{n} t_{m}} \bar{x}_{m} \\
x_{n} \geq 0
\end{array}\right.
$$

This linear program has a solution for dual completion $\bar{\pi}_{n}$ obtained from fully integrated problem is feasible for Linear Program. Therefore, it can be used $\bar{x}_{1},\left\{\bar{\pi}_{n}\right\}_{n \in \mathcal{N}}$ dan $\left\{\mathcal{B}_{n}\right\}_{n \in \mathcal{N}}$ to produce a policy against initial problem. This is obtained by using the equation,

$$
\begin{gathered}
x_{2, B}(\vec{\xi})=\mathcal{B}_{2}^{-1}\left(b_{2}\left(\vec{\xi}_{2}\right)-A_{21} \hat{x}_{1}\right) \\
x_{2, N}(\vec{\xi})=0 \\
x_{t_{n}, B}(\vec{\xi})=\mathcal{B}_{n}^{-1}\left(b_{t_{n}}\left(\vec{\xi}_{t_{n}}\right)-\sum_{r=1}^{t_{n}-1} A_{t_{n}{ }^{\prime}} x_{r}\left(\vec{\xi}_{r}\right)\right) \\
x_{t_{n}, N}(\vec{\xi})=0
\end{gathered}
$$

\subsection{Completion Method}

Basic design model is in the form of 


$$
\begin{array}{lll}
\text { Min } & F(\underline{x})+\underline{d}^{T} \underline{y} & \\
\text { Constrains } & f(\underline{x})+A_{1} \underline{y}=\underline{b}_{1} & \left(m_{1} \text { row }\right) \\
& A_{2} \underline{x}+A_{3} \underline{y}=\underline{b}_{2} & \left(m_{2} \text { row }\right) \\
& \underline{\ell} \leq(\underline{x}, \underline{y})^{T} \leq \underline{u} &
\end{array}
$$

The algorithm takes place by working row major iteration, in which the constraints is in linierisation at some point of the line $\underline{x}_{k}$ and nonlinearitas coupled with the objective function along with estimates of the Lagrange multiplier.

So, $\hat{f}\left(\underline{x}, \underline{x}_{k}\right)=f\left(\underline{x}_{k}\right)+J\left(\underline{x}_{k}\right)\left(\underline{x}-\underline{x}_{k}\right)$. Then the subproblems which have linear constraints, resolved on major iteration $k^{\text {th }}$, is:

$$
\begin{array}{rl}
\min _{\underline{x}, \underline{y}} & L\left(\underline{x}, \underline{y}, \underline{x}_{k}, \lambda_{k}, \rho\right)=F(\underline{x})+\underline{d}^{T} \underline{y} \\
& -\lambda_{k}^{T}(f-\hat{f})+\frac{1}{2} \rho(f-\hat{f})^{T}(f-\hat{f})
\end{array}
$$

constrains $\partial_{k} \underline{x}+A_{1} \underline{y} \mp \underline{b}_{1}+J_{k} \underline{x}_{k}-f\left(\underline{x}_{k}\right)$

$$
\begin{aligned}
& A_{2} \underline{x}+A_{2} \underline{y}=\underline{b}_{2} \\
& \underline{\ell} \leq(\underline{x}, \underline{y})^{T} \leq \underline{u}
\end{aligned}
$$

Objective function is an extension of the modified Lagrange, penalty parameter $\rho$ accelerate the convergence of initial estimates point to be away from the optimal point. Lagrange multiplier $\lambda_{k}$ taken as the optimal value on completion of the previous sub-problems. If the main iteration row approaching the optimum point (measured by the relative change in estimate $\lambda_{k}$ and the degree to which non linear constraints are met $\underline{x}_{k}$ ), penalty parameter $\rho$ reduced to be 0 . The proposed method uses a strategy of active constraints, ie

$$
A \underline{x}=\left[\begin{array}{ccc}
B & S & N \\
& & I
\end{array}\right]\left[\begin{array}{l}
\underline{x}_{B} \\
\underline{x}_{S} \\
\underline{x}_{N}
\end{array}\right]=\left[\begin{array}{c}
\underline{b} \\
- \\
b_{N}
\end{array}\right]
$$

$B$ the set of basis vectors

$S$ the set of superbasis vectors

$N$ the set of nonbasis vectors

$I$ matrix unit

Nonbasis variable $\underline{x}_{N}$ is at the limit and remain there for a next step $\Delta \underline{x}$. Based on this is obtained, $B \underline{x}_{B}+S \underline{x}_{S}+N \underline{x}_{N}=\underline{b}$

$$
\underline{x}_{N}=\underline{b}_{N} \text {, with } \underline{b}_{N} \text { is a combination of upper }
$$

limit and lower limit. Independent superbasis variable $\underline{x}_{S}$ move in any direction and give a boost to minimize the objective function. Basis $\underline{x}_{B}$ should follow the equation $B \underline{x}_{B}+S \underline{x}_{S}=0 . \Delta x$ can be written in a change in superbasis variables, namely $\Delta \underline{x}=Z \Delta \underline{x}_{S}$, with $Z=\left[\begin{array}{c}-B^{-1} S \\ I \\ 0\end{array}\right]$
Matrix Z works as a 'reduction' matrix and multiplying from the left of gredien vectors to form a gradient of reduced $\underline{h}=Z^{T} \underline{g}$ with $\underline{g}=\partial \ell / \partial \underline{x}$. And also multiplying from the left and right Hessi matrix of The second partial derivatives to produce steps such Newton in the reduced space of superbasis variables.

The implementation of this method use approximation of quasi-Newton $R^{T} R$ of the reduced Hessi matrix, which $\mathrm{R}$ is the upper triangular matrix. 'Sparsity' within the constraints maintained by storing and updating the factorization $L U$ of $\mathrm{B}$ Basis matrix.

This factorization gives the sense that $Z$ or $B^{-1}$ not stated explicitly. Step of quasi-Newton $\Delta \underline{x}$ calculated with the following sequence :

i) Solve $U^{T} L^{T} \bar{a}=g_{B}$ for $\bar{a}$ where gradient vector $\underline{g}$ partitioned into $\left(\underline{g}_{B}, \underline{g}_{S}, \underline{g}_{N}\right)$ related to the partition $A$ and $\Delta \underline{x}$

ii) Form $\underline{h}=\underline{g}_{S}-S^{T} \bar{a}$

iii) Solve $R^{T} R \Delta \underline{x}_{S}=-\underline{h}$

iv) Solve $L U \Delta \underline{x}_{B}=-S \Delta \underline{x}_{S}$, superbasis set size varies when the search algorithm progresses.

If the limit variables encountered, the variables made into nonbasis and moved from the set superbasis (or basis). Whereas if convergence is achieved in a subspace, one or more nonbasis variables be made superbasis if element vector 'reduced cost' linked of $\underline{g}_{N}-N^{T} \bar{a}$ not zero and appropriate.marked.

The previous section are methods / algorithms to solve stochastic linear program and not linear. Based on the framework of the method is developed mixture chopped stochastic program.

\subsection{Algoritma Dari Metode}

After finishing the relaxation problem, the search procedure completion of chopped region is described, $x=[x]+f, \quad 0 \leq f<1$. The completion of the continuous relaxation problem is with the following stages.

Step 1: Choose the basis $i^{*}$ is the smallest chopped infisiclity, so that $\delta_{i^{*}}=\min \left\{f_{i}, 1-f_{i}\right\}$

Step 2: Do operation of pricing, ie count $v_{i^{*}}^{T}=\ell_{i^{*}}^{T} B^{-1}$

Step 3: Calculate $\sigma_{i j}=v_{i^{*}}^{T} a_{j}$ with $\mathrm{j}$ linked with $\min _{j}\left\{\left|\frac{\ell_{i}}{\sigma_{i j}}\right|\right\}$

I. For non basis $\mathrm{j}$ at lower limit

If $\sigma_{i j}<0$ and $\delta_{i^{*}}=f_{i}$ calculate $\Delta=\frac{\left(1-\delta_{i^{*}}\right)}{-\sigma_{i j}}$

If $\sigma_{i j}>0$ and $\delta_{i^{*}}=1-f_{i}$ calculate $\Delta=\frac{\left(1-\delta_{i^{*}}\right)}{\sigma_{i j}}$

If $\sigma_{i j}<0$ and $\delta_{i^{*}}=1-f_{i}$ calculate $\Delta=\frac{\delta_{i^{*}}}{-\sigma_{i j}}$ 
If $\sigma_{i j}>0$ and $\delta_{i^{*}}=f_{i}$ calculate $\Delta=\frac{\delta_{i^{*}}}{\sigma_{i j}}$

II. For non basis $\mathrm{j}$ at upper limit

If $\sigma_{i j}<0$ and $\delta_{i^{*}}=1-f_{i}$ calculate $\Delta=\frac{\left(1-\delta_{i^{*}}\right)}{-\sigma_{i j}}$

If $\sigma_{i j}>0$ and $\delta_{i^{*}}=f_{i}$ calculate $\Delta=\frac{\left(1-\delta_{i^{*}}\right)}{\sigma_{i j}}$

If $\sigma_{i j}>0$ and $\delta_{i^{*}}=1-f_{i}$ calculate $\Delta=\frac{\delta_{i^{*}}}{\sigma_{i j}}$

If $\sigma_{i j}<0$ and $\delta_{i^{*}}=f_{i}$ calculate $\Delta=\frac{\delta_{i^{*}}}{-\sigma_{i j}}$

If not, go to the non-base or next $\mathrm{j}$ superbasis (if any). So $j^{*}$ column raised from the lower limit or bound from the upper limit. If no, go to the next $i^{*}$.

Step 4: Calculate $\quad \alpha_{j^{*}}=B^{-1} a_{j^{*}} \quad$ ie, solve $B \alpha_{j^{*}}=a_{j^{*}}$ untuk $\alpha_{j^{*}}$

Step 5: Feasibility test, There are three possibilities for the variables fixed base. Eligible because of release nonbasis $j^{*}$ variable from it's limit.

$\rightarrow$ if $j^{*}$ at lower limit.

Take

$$
\begin{aligned}
& A=\operatorname{Min}_{i^{\prime} \neq i^{*} \mid \alpha_{i^{*}}>0}\left\{\frac{x_{B_{i^{\prime}}}-\ell_{i^{\prime}}}{\alpha_{i j^{*}}}\right\} \\
& B=\operatorname{Min}_{i^{\prime} \neq i^{*} \mid \alpha_{i^{*}}<0}\left\{\frac{a_{i^{\prime}}-x_{B_{i^{\prime}}}}{-\alpha_{i j^{*}}}\right\} \\
& C=\Delta
\end{aligned}
$$

Maximum motion of $j^{*}$ depending on $\theta^{*}=\min (A, B, C)$

$\rightarrow$ if $j *$ at upper limit

Take

$$
\begin{aligned}
& A^{\prime}=\operatorname{Min}_{i^{\prime} \neq i^{*} \mid \alpha_{i j^{*}}>0}\left\{\frac{x_{B_{i^{\prime}}}-\ell_{i^{\prime}}}{-\alpha_{i j^{*}}}\right\} \\
& B^{\prime}=\operatorname{Min}_{i^{\prime} \neq i^{*} \mid \alpha_{i^{*}}<0}\left\{\frac{a_{i^{\prime}}-x_{B_{i^{\prime}}}}{\alpha_{i j^{*}}}\right\} \\
& C^{\prime}=\Delta
\end{aligned}
$$

Maximum motion of $j *$ depending on

$$
\theta^{*}=\min \left(A^{\prime}, B^{\prime}, C^{\prime}\right)
$$

Step 6 Exchange basis for all 3 possibilities 1. If $A$ or $A^{\prime}$

- $\quad x_{B_{i^{\prime}}}$ become nonbasis at lower limit $\ell_{i^{\prime}}$

- $\quad x_{j^{*}}$ become basis (replace $x_{B_{i^{\prime}}}$ )

- $\quad x_{i^{*}}$ fixed basis (not chopped).

2. If $B$ or $B$,
- $\quad x_{B_{i^{\prime}}}$ become nonbasis at upper limit $a_{i}$

- $\quad x_{j^{*}}$ become basis (replace $x_{B_{i^{\prime}}}$ )

- $\quad x_{i^{*}}$ fix basis (not chopped).

3. If $C$ or $C^{\prime}$

- $\quad x_{j^{*}}$ become basis (replace $x_{i^{*}}$ )

- $x_{i^{*}}$ become superbasis worth chopped

\section{Results}

The program is built is for two raw materials, namely material 1 and material 2. From the raw materials produced two different production is the production of 1 and 2 . The production cost per unit of production of raw materials is expressed as the unit cost of the raw materials $c=\left(c_{\text {materiall }}\right.$, $\left.c_{\text {material } 2}\right)^{T}$, the need for production $h=\left(h_{\text {prod } 1}, h_{\text {prod } 2}\right)^{T}$ and the production capacity is $\hat{b}$. The maximum total amount of raw materials that can be processed, given in Table 1 .

Table 1. Productivit $\pi$ (material i, product $j$ ).

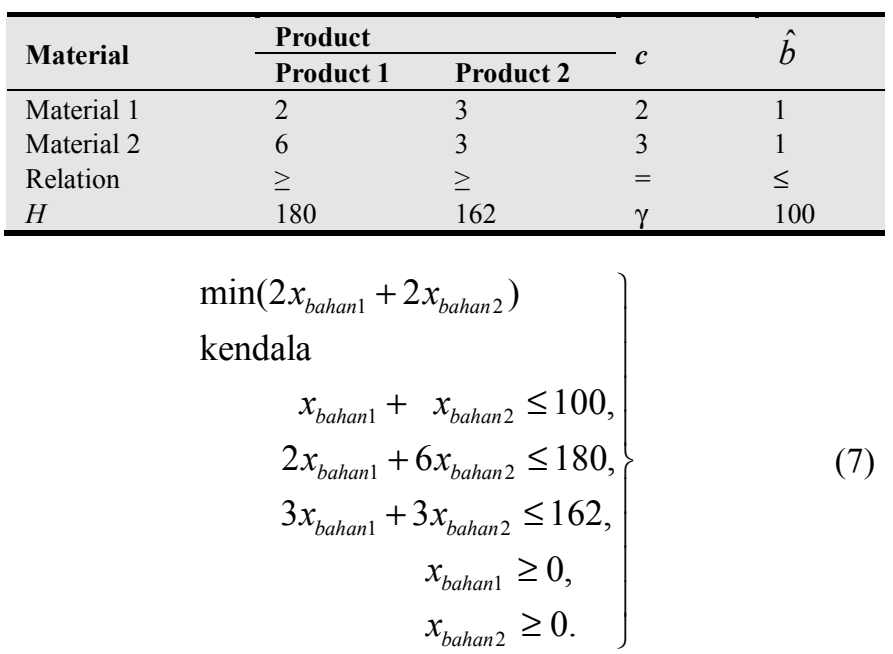

By using graphs, be obtained completion

$$
\hat{x}_{\text {bahan } 1}=36, \hat{x}_{\text {bahan } 2}=18, \gamma(\hat{x})=126
$$

which is a single optimal solution to the above problem.

The above issues are described by (7) and solved by (8) with the requirements of productivity, cost per unit, needs and capacities (Table 1), with fixed data and know-making with production planning. It often happens, some of the dataproductivity and needs changing (random). As a result, production planning decisions can not be made because of data are not exact values.

If it is assumed statistically, it is known that:

$$
\left.\begin{array}{r}
h_{\text {product } 1}=180+\tilde{\zeta}_{1}, \\
h_{\text {product } 1}=160+\tilde{\zeta}_{2}, \\
\pi\left(\text { material1, material2) }=2+\tilde{\eta}_{1}\right. \\
\pi\left(\text { material1, material2) }=3.4+\tilde{\eta}_{2}\right.
\end{array}\right\}
$$


which $\tilde{\zeta}_{j}$ is a random variable, is modeled by using a normal distribution, $\tilde{\eta}_{1}$ dan $\tilde{\eta}_{2}$ is the uniform distribution and exponential distribution. With parameters:

$$
\left.\begin{array}{l}
\operatorname{dist} \tilde{\zeta}_{1} \sim N(0,12) \\
\operatorname{dist} \tilde{\zeta}_{2} \sim N(0,9) \\
\operatorname{dist} \tilde{\eta}_{1} \sim U(-0.8,0.8) \\
\operatorname{dist} \tilde{\eta}_{2} \sim \operatorname{Exp}(\lambda=2.5)
\end{array}\right\}
$$

For simplicity, it is assumed that the four random variables are mutually independent, because of the random variable $\tilde{\zeta}_{1}, \tilde{\zeta}_{2}$ dan $\tilde{\eta}_{2}$ is not limited, restricted $99 \%$ confidence intervals (except $\mu$ ). In order to obtain a random variable realization

$$
\left.\begin{array}{l}
\tilde{\zeta}_{1} \in\left[\begin{array}{ll}
-30.91, & 30.91
\end{array}\right] \\
\tilde{\zeta}_{2} \in\left[\begin{array}{ll}
-23.18, & 23.18
\end{array}\right] \\
\tilde{\eta}_{1} \in\left[\begin{array}{ll}
-0.8,0.8
\end{array}\right] \\
\tilde{\eta}_{2} \in\left[\begin{array}{ll}
0.0, & 1.84
\end{array}\right]
\end{array}\right\}
$$

In place of a linear program (7), given stochastic linear program

$$
\min \left(2 x_{\text {material } 1}+2 x_{\text {material } 2}\right)
$$

constrains

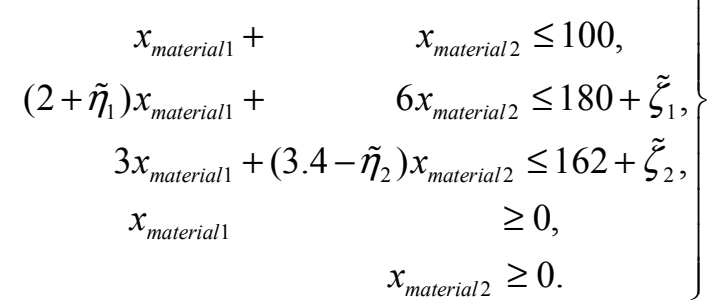

Issue a decision on the above, are not defined because everything is not clear whether the "min" can be obtained before the realization $\left(\zeta_{1}, \zeta_{2}, \eta_{1}, \eta_{2}\right)$ from $\left(\tilde{\zeta}_{1}, \tilde{\zeta}_{2}, \tilde{\eta}_{1}, \tilde{\eta}_{2}\right)$ known. For comparison, the deterministic problem of the issues described above is $\hat{x}=\left(\hat{x}_{\text {material } 1}, \hat{x}_{\text {material } 2}\right)=(36,18), \gamma=126$. Production planning is

$$
\left.\begin{array}{l}
\hat{y}=\left(\hat{y}_{\text {material } 1}, \hat{y}_{\text {material } 2}\right)=(20,30), \gamma=130, \\
\hat{z}=\left(\hat{z}_{\text {material } 1}, \hat{z}_{\text {material } 2}\right)=(50,22), \gamma=166, \\
\hat{v}=\left(\hat{v}_{\text {material } 1}, \hat{v}_{\text {material } 2}\right)=(58,6), \gamma=134
\end{array}\right\}
$$

which is a solution wait and see.

The "wait and see" solutuion not necessary because the production plan contains uncertainties that only provide information about the needs and random productivity. More practical, can be determined production plan, to minimize the total cost of the early first phase (ie production) and average recourse cost instead of the random variable $\tilde{\zeta}_{1}, \tilde{\zeta}_{2}, \tilde{\eta}_{1}$, dan $\tilde{\eta}_{2}$, is used random vector notation $\tilde{\xi}=\left(\tilde{\zeta}_{1}, \tilde{\zeta}_{2}, \tilde{\eta}_{1}, \tilde{\eta}_{2}\right)^{T}$. Furthermore, introduced to each stochastic constraints on (7) one recourse variable $y_{i}(\tilde{\xi}), i=1,2$.

The stochastic program at (7) will be converted into a stochastic program with recourse, using

$$
\begin{aligned}
& h_{1}(\tilde{\xi}):=h_{\text {product } 1}=180+\tilde{\zeta}_{1}, \\
& h_{2}(\tilde{\xi}):=h_{\text {product } 2}=162+\tilde{\zeta}_{2}, \\
& \alpha(\tilde{\xi}):=\pi(\text { material } 1, \text { product } 1)=2+\tilde{\eta}_{1} \\
& \beta(\tilde{\xi}):=\pi(\text { material } 2, \text { product } 2)=3.4+\tilde{\eta}_{2}
\end{aligned}
$$

$$
\left.\begin{array}{rr}
\min \left\{2 x_{a 1}+3 x_{\text {material } 2}+E_{\tilde{\xi}}\left[7 y_{1}\right.\right. & \\
\text { constrains } x_{\text {material } 1}+\quad x_{\text {material } 2} & \leq 100, \\
\alpha(\tilde{\xi}) x_{\text {material } 1}+\quad 6 x_{\text {material } 2}+y_{1}(\tilde{\xi}) & \geq h_{1}(\tilde{\xi}), \\
3 x_{\text {material } 1}+\beta(\tilde{\xi}) x_{\text {material } 2}+y_{2}(\xi) & \geq h_{1}(\tilde{\xi}), \\
x_{\text {material } 1} & \geq 0 \\
x_{\text {material } 2} & \geq 0 \\
y_{1}(\tilde{\xi}) & \geq 0 \\
& y_{2}(\tilde{\xi}) \\
& \geq 0
\end{array}\right\}
$$

If $\quad \tilde{\xi}$ has finite discrete distribution $\left\{\left(\xi^{i}, p_{i}\right), i=1, \ldots, r\right\}$ and $\left(p_{i} \geq 0, \forall i\right)$ so problema of (14) become linear program, known as dual decomposition structure

$$
\left.\begin{array}{rr}
\min \left\{2 x_{\text {material } 1}+3 x_{\text {material } 2}+\sum_{i=1}^{r} p_{i}\left[7 y_{1}\left(\xi^{i}\right)+12 y_{2}\left(\xi^{i}\right)\right]\right\} & \leq 100, \\
\text { constrains } \quad x_{\text {material } 1}+\quad x_{\text {material } 2} & h_{1}\left(\xi^{i}\right) \forall i, \\
\alpha\left(\xi^{i}\right) x_{\text {material } 1}+\quad 6 x_{\text {material } 2}+y_{1}\left(\xi^{i}\right) & \geq 0, \\
3 x_{\text {material } 1}+\beta\left(\xi^{i}\right) x_{\text {material } 2}+y_{2}\left(\xi^{i}\right) \geq & h_{1}\left(\xi^{i}\right) \forall i, \\
x_{\text {material } 1} & \geq 0, \\
x_{\text {material } 2} & \geq 0 \forall i, \\
y_{1}\left(\xi^{i}\right) \quad & \forall i .
\end{array}\right\}
$$

The solution of problema (15) by using the proposed method, we get the solution of $\bar{x}$,

$$
\bar{x}=(37.566,22.141), \quad \gamma(\bar{x})=144.179, \quad \gamma_{I}(\bar{x})=141.556,
$$

Where the solution of linear program (6.1) produce a total average cost $\gamma(\bar{x})=204.561$ with reliability obtained is $\rho(\bar{x})=0.9497$, with a difference $\rho(\hat{x})=0.2983$ in solution of linear program $\hat{x}$. 


\section{Conclusion}

Stochastic optimization program associated with decisionmaking the uncertainty of the data problems from time to next time. Type object of study is a random optimization problem where the outcomes of random data is not revealed at run time, and decisions to be optimized not have to anticipate future results (non-anticipation). Provided probabilistic information available, the appropriate operational models for optimization, can be formulated as a dual-stage stochastic program. This model essentially proposed to replace the deterministic model, where the coefficients or parameters are not known to be random by assuming the distribution of free opportunities of the decision variables. But to accomplish this stochastic programming model needs to be reformulated into an equivalent deterministic program. The scale of dimensional deterministic program will 'explode' because the number of scenarios that are so profound. Therefore, it is also necessary that the scenario forming technique 'explosion' can be avoided.

\section{Acknowledgements}

The authors thank to Hamonangan Tambunan, Lecturer of Faculty of Engineering, State University of Medan, for his assistance to the author.

\section{References}

[1] Klein-Haneveld, W.K., L. Stougie and M.H. van der Vlerk. 1996. An Algorithm for the Construction of Convex Hulls in Simple Integer Recourse Programming, Annals of Operational Research, 64, 67-81. http://dx.doi.org/10.1007/bf02187641.

[2] Laporte, G., and F.V. Louveaux, 1993. The Integer L-shaped Method for Stochastic Integer Programs with Complete Recourse. Operations Research Letters, 13 133-142. http://dx.doi.org/10.1016/0167-6377(93)90002-x.

[3] Sen, S., and J.L. Higle. 2003. The $C^{3}$ Theorem and a $D^{2}$ Algorithma for Large Scale Stochastic Integer Programming. Set Convexification. Working Paper Univ. of Arizona. http://dx.doi.org/10.1007/s10107-004-0566-z.

[4] Sherali, Hanif D, and Fraticelli, Barbara M. P. 2002. A modification of Benders' decomposition algorithm for discrete subproblems: An approach for stochastic programs with integer recourse. Journal of Global Optimization. 22(1-4), pp 319-342. http://dx.doi.org/10.1023/a:1013827731218.

[5] Caroe,C.C., and R.Schultz. 1999. Dual Decomposition in Stochastic Integer Programming. Oper. Res. Lett. 24, 37-45. http://dx.doi.org/10.1016/s0167-6377(98)00050-9.

[6] Rockafellar, R. T. and . Wets, Roger J.-B. 1991. Scenarios and Policy Aggregation in Optimization Under Uncertainty. Mathematics of Operations Research, 16(1), 1991, 119-147. http://dx.doi.org/10.1287/moor.16.1.119

[7] Ahmed, M. B. et al. 2002. Ex-ante impact assessment and economic analysis of breeding for nutrient efficiency and alternate strategies, a conceptual model and research issues: A socio-economist's view. Food Security in Nutrient-Stressed Environments: Exploiting Plants' Genetic Capabilities on 3742. http://dx.doi.org/10.1007/978-94-017-1570-6_5.

[8] Takriti, S., J.R.Birge., and E.Long. 1996. A Stochastic Model for the Unit Commitment Problem. IEEE Trans. Power Syst. 11, 1497-1508. http://dx.doi.org/10.1109/59.535691.

[9] Schultz, R. L.Stougie, and M. H. van der Vlerk. 1998. Solving Stochastic Programs with Integer Recourse by Enumeration: A framework using Grobner Basis Reductions. Math. Programming, 83, 229-252. http://dx.doi.org/10.1007/bf02680560.

[10] Erlinawaty and H. Mawengkang. 2006. A Feasible Neighborhood search for Portfolio Optimization Problems, Proceedings of Int. Conference of IRMSA, Penang, Malaysia.

[11] Lokketangen, A., and D.L. Woodruff, 1993, Progressive Hedging and Tabu Search Applied to Mixed Integer $(0,1)$ Multi-stage Stochastic Programming, J. of Heuristics, 2 ,111128. http://dx.doi.org/10.1007/bf00247208.

[12] Caroe,C.C., and R.Schultz. 1999. Dual Decomposition in Stochastic Integer Programming. Oper. Res. Lett. 24, 37-45. http://dx.doi.org/10.1016/s0167-6377(98)00050-9.

[13] Schultz, R.; Tiedemann, S., 2003. Risk Aversion via Excess Probabilities in Stochastic Programs with Mixed-Integer Recourse. SIAM J.Optim. 14, 115-138. http://dx.doi.org/10.1137/s1052623402410855.

[14] Lulli, G.,and S. Sen. 2003. A Branch and Price Algorithm for Multi-Stage Stochastics Integer Programming with Application to Stochastic Batch-Sizing Problems. Working Paper, Univ. of Arizona. http://dx.doi.org/10.1287/mnsc.1030.0164.

[15] Huang et al. 2005. Green's Function Analysis Of An Ideal Hard Surface Rectangular Waveguide. Radio Science, 40(5),2005. Http://Dx.Doi.Org/10.1029/2004rs003161.

[16] Rubinstein, Reuven Y. and Shapiro, Alexander. 1990. Optimization of static simulation models by the score function method. Mathematics and Computers in Simulation, 32(4), 373-392. http://dx.doi.org/10.1016/0378-4754(90)90142-6.

[17] Plambeck et al. (1996). Sample-path optimization of convex stochastic performance functions. Mathematical Programming, 75(2), 137-176. http://dx.doi.org/10.1016/s00255610(96)00010-x.

[18] Goyal Vishal, M.G. Ierapetaiton. 2007. Stochastic MINLP Optimization using simplicial appreoximation, Computers and Chemical Engineering, vol. 31, pp. 1081-1087. http://dx.doi.org/10.1016/j.compchemeng.2006.09.013.

[19] Goyal, Vishal Marianthi, Ierapetritou G. . 2004. Framework for evaluating the feasibility/operability of onconvex processes. AIChE Journal. 49(5). 1233- 1240. http://dx.doi.org/10.1002/aic.690490514.

[20] Diwekar, U. 2003a. Greener by design, Environmental Science and Techology, 37, 5432-5444. http://dx.doi.org/10.1021/es0344617.

[21] Mohit Tawarmalani, Nikolaos V. Sahinidis. 2004. Global optimization of mixed-integer nonlinear programs: A theoretical and computational study. Mathematical Programming. 99 (3). 563-591. http://dx.doi.org/10.1007/s10107-003-0467-6. 
[22] Olsen, C F. 1976. Digital computation of inverse Laplace transform. SIMULATION, 27(6), 197- 201 http://dx.doi.org/10.1177/003754977602700605. 